\title{
Expression and function of aquaporins in peripheral nervous system
}

\author{
Tong-hui $\mathrm{MA}^{1, *}{ }^{*}$, Hong-wen $\mathrm{GAO}^{1}$, Xue-dong FANG ${ }^{1}$, Hong YANG ${ }^{2}$ \\ ${ }^{1}$ Central Research Laboratory, Jilin University Bethune Second Hospital, Changchun 130041, China; ${ }^{2}$ School of Life Sciences, Liaoning \\ Provincial Key Laboratory of Biotechnology and Drug Discovery, Liaoning Normal University, Dalian 116029, China
}

The expression and role of the aquaporin (AQP) family water channels in the peripheral nervous system was less investigated. Since 2004, however, significant progress has been made in the immunolocalization, regulation and function of AQPs in the peripheral nervous system. These studies showed selective localization of three AQPs (AQP1, AQP2, and AQP4) in dorsal root ganglion neurons, enteric neurons and glial cells, periodontal Ruffini endings, trigeminal ganglion neurons and vomeronasal sensory neurons. Functional characterization in transgenic knockout mouse model revealed important role of AQP1 in pain perception. This review will summarize the progress in this field and discuss possible involvement of AQPs in peripheral neuropathies and their potential as novel drug targets.

Keywords: aquaporins; peripheral nervous system; neuronal transduction; gene knockout; gene expression; pain

Acta Pharmacologica Sinica (2011) 32: 711-715, doi: 10.1038/aps.2011.63; published online 23 May 2011

\section{Introduction}

Aquaporins (AQPs) are a family of water-transporting proteins selectively expressed in epithelial, endothelial and many other cell types where they play important physiological functions ${ }^{[1,2]}$. In the past two decades, studies on the expression and function of AQPs in the nervous system have been focused mainly in the central nervous system. Numerous studies reported the localization of six AQPs including AQP1, AQP3, AQP4, AQP5, AQP8, and AQP9 in the brain and spinal $\operatorname{cord}^{[3]}$. Functional studies using transgenic knockout mouse model revealed important roles of AQP1 in cerebral spinal fluid (CSF) secretion and pain sensation and AQP4 in brain edema formation, neuronal transduction, neurogenesis, glioma spreading, neuroinflammation and pain perception ${ }^{[3-12]}$.

In contrast, the expression and function of AQPs in the peripheral nervous system are less investigated. Until 2004, Matsumoto et al first described AQP1 mRNA expression in the trigeminal ganglion by the hierarchical cluster analysis of DNA microarray and in situ hybridization ${ }^{[13]}$. Since then, significant progress has been made in the immunolocalization, regulation and function of AQPs in the peripheral nervous system. So far studies have reported the expression of three AQPs including AQP1, AQP2, and AQP4 in peripheral neuronal or glial elements such as dorsal root ganglion neu-

\footnotetext{
* To whom correspondence should be addressed.

E-mail math108@gmail.com

Received 2011-03-16 Accepted 2011-04-20
}

rons, enteric neurons and glial cells, periodontal Ruffini endings, trigeminal ganglion neurons and vomeronasal sensory neurons. Functional characterization in transgenic knockout mouse model suggested important role of AQP1 in peripheral pain perception. This review will summarize the progress in this field and discuss possible involvement of AQPs in peripheral neuropathies and their potential as novel drug targets.

\section{Expression and function of AQP1 in peripheral sensory nerves}

In a study to identify candidate genes involved in somatosensory functions of cranial sensory ganglia reported in 2004, Matsumoto et al first described the expression of AQP1 mRNA in neurons of somatosension-related ganglia by the hierarchical cluster analysis of DNA microarray ${ }^{[13]}$. Further analysis by in situ hybridization showed specific expression of AQP1 mRNA in neurons with higher frequencies in trigeminal and petrosal ganglion and with low frequency in nodose ganglia. AQP1 is expressed in small to medium size neurons with a diameter under $30 \mu \mathrm{m}$. The expression pattern suggested the involvement of AQP1 in somatosensation in cranial structures such as the face, oral cavity and pharynx. Although there was no protein expression data provided, this is the first report of an AQP expression in peripheral nerve.

Later on, a study conducted by Oshio et al reported AQP1 protein localization in nerve fibers in the trigeminal and sciatic nerves as well as in small neurons and nerve fibers in dorsal root ganglia (DRG) in the peripheral nervous system ${ }^{[14]}$. Co- 
localization of AQP1 with substance P and capsaicin receptor TRPV1 in DRG suggested that AQP1 was expressed in $\mathrm{C}$-fiber nociceptive neurons and possibly contributed to the processing of pain signals. They performed a study on the role of AQP1 in pain sensation by comparing acute pain responses to thermal, mechanical, and chemical noxious stimuli in AQP1 knockout (AQP1 ${ }^{-/-}$) and wildtype (AQP1 ${ }^{+/+}$) mice. Although no significant differences were found in the morphology or number of neurons expressing SP or TRPV1 in the DRG between $\mathrm{AQP1} 1^{-/-}$and $\mathrm{AQP1} 1^{+/+}$mice, they showed that $\mathrm{AQP1} 1^{-/-}$mice had reduced responsiveness to thermal and capsaicin chemical stimuli, but not to mechanical stimuli or formalin ${ }^{[14]}$. This study was the first evidence for a role of AQP1 in pain signal transduction.

However, a following study reported by Shields et al was in marked disagreement with the above functional data ${ }^{[15]}$. By more sophisticated expression analysis, they found several lines of evidences suggesting the involvement of AQP1 in pain sensation including: (1) AQP1 protein is present in the spinal cord in laminae associated with nociceptive processing, (2) AQP1 is present on the membrane of DRG neurons neurochemically defined as nociceptors, (3) the onset of AQP1 expression in DRG corresponds temporally to the formation of functional synaptic contacts between nociceptors and their postsynaptic partners in the spinal cord, and (4) AQP1 labeling in the dorsal horn changes after sciatic nerve lesion, a manipulation that results in an altered pain state. They then performed in vivo electrophysiological measurements and behavioral analyses in a comprehensive battery of acute and persistent pain tests on $\mathrm{AQP1} 1^{-/}$and $\mathrm{AQP} 1^{+/+}$mice to evaluate a functional role of AQP1 in nociceptive processing. Unexpectedly, they could not detect a differential phenotype suggesting a functional contribution of AQP1 to nociceptive processing in all the acute and persistent pain tests including Hargreaves test, tail flick test, Hot plate test, von Frey test of mechanical threshold, spared nerve injury model of neuropathic pain, capsaicin test, prostaglandin E2 inflammation and hypoosmolar challenge. They concluded that AQP1 was not required for normal pain processing despite its abundant and restricted expression in nociceptive primary afferent neurons.

To resolve the discrepancy for the role of AQP1 in pain physiology, Zhang and Verkman performed a study with more extensive behavioral testing as well as immunolocalization, water permeability, and patch clamp studies on freshly isolated, dissociated DRG neurons from $\mathrm{AQP1}^{-/-}$and $\mathrm{AQP1} 1^{+/+}$ mice ${ }^{[16]}$. They reported greatly reduced behavioral responses to inflammatory thermal and cold pain in litter-matched $\mathrm{AQP1}^{-/-}$mice. In mechanistic studies, they found distinct electrophysiological defects related to impaired $\mathrm{Na}_{\mathrm{v}} 1.8 \mathrm{Na}^{+}$ channel functioning in AQP1-deficient DRG neurons. By patch clamp, immunoprecipitation, and single particle tracking studies in transfected cell models, the researchers identified a novel AQP1- $\mathrm{Na}_{\mathrm{v}} 1.8$ interaction that may be responsible, in part, for the impaired pain sensation in AQP1 deficient mice. Based on these results, the researchers proposed potential utility of AQP1 inhibitor to reduce pain nociception as a novel strategy to achieve analgesia at the presynaptic spinal level.

It is not known why these studies generated conflict results in the same AQP1 knockout mouse model by similar approaches. More investigations will be required to establish a role of AQP1 in peripheral pain perception. Based on some recent studies that indicated functional involvement of AQP1 and AQP4 in pain perception of the central nervous system $^{[10-12]}$, we have reason to speculate a contribution of AQP1 in certain types of peripheral pain perception.

\section{Expression of AQP1 in enteric nervous system}

Gao et al first reported the expression pattern of AQP1 in human enteric nervous system by immunolocalization in $2006^{[17]}$. Strong AQP1 expression was identified in the submucosal and myenteric nerve plexuses in the esophagus. AQP1 was localized to the same cell population expressing glial fibrillary acidic protein (GFAP), but clearly not to the neurons in the ganglia, indicating glial cell-specific expression. The same study also described glial selective expression of AQP1 in pancreatic ganglia. AQP4 and AQP9, which are broadly expressed in astroglial cells in brain and spinal cord, were not localized in glial cells or neurons in the peripheral nerve plexuses. The study concluded that AQPs were differentially expressed in the peripheral versus central nervous system and that channel-mediated water transport mechanisms may be involved in peripheral neuronal activity by regulating water homeostasis in the ganglia and nerve fiber bundles.

Some following studies indicated that the expression of AQP1 in the enteric nervous system exhibited species difference. Nagahama et al reported the localization of AQP1 protein in a particular neuronal subtype in the enteric nervous system of the rat ileum ${ }^{[18]}$. Co-localization study indicated that AQP1-positive neurons simultaneously expressed a neuronal marker HuC/D. AQP1 is expressed in a subpopulation $(\sim 9.3 \%)$ of the $\mathrm{HuC} / \mathrm{D}$-positive neurons. AQP1-positive neurons were classified as Dogiel type I cells, which have several short processes and a single long process. Many AQP1-positive nerve fibers were also found both in the myenteric and submucosal plexuses in all regions of the ileum. The neuronal expression pattern of AQP1 in rat ileum is apparently different from the glial cell-specific expression seen in the nerve plexuses in human esophagus and pancreas ${ }^{[17]}$.

In consistent with the above study, Ishihara et al confirmed the localization of AQP1 in neurons and fibers of rat myenteric plexus ${ }^{[19]}$. Based on their analysis, AQP1 is expressed in a subpopulation (about 5.5\%) of the HuC/D-positive neurons, slightly different from the data by Nagahama et $a l^{[18]}$. To study the possible involvement of AQP1 in diabetic gastrointestinal dysfunctions, the researchers examined the pathological changes in AQP1-positive neurons in streptozotocin-induced (STZ) diabetic rats. Although the total number of the $\mathrm{HuC} /$ D-positive neurons is similar between normal and STZ rats, AQP1-positive neurons in STZ rats were found to be significantly increased by two-fold ( $11.2 \%$ vs $5.5 \%$ in normal rats). It was also described that many AQP1-positive fibers with 
swollen varicosities were seen in the secondary and tertiary myenteric plexus and in the longitudinal and circular muscle layers of STZ rats. The swollen AQP1-positive varicosities and increased AQP1-positive neurons were thought to be the consequence of the long-term diabetic conditions in STZ rats. It was postulated that these pathological changes of AQP1-positive neurons possibly contribute to diabetic gastrointestinal dysfunction in streptozotocin-induced diabetic rats. Therefore, AQP1 may become a potential new therapeutic target for gastrointestinal dysfunction in diabetes.

Recently, another study by Arciszewski et al supported the presence of AQP1 in enteric neurons ${ }^{[20]}$. However, their results indicated AQP1 expression on submucosal but not myenteric neurons in the sheep duodenum. The vast majority of AQP1bearing submucosal neurons were immunoreactive to substance $P$, suggesting that they are probably a subpopulation of sensory neurons. In both the rat and sheep studies, AQP1 expression was not detected in glial elements of submucosal plexus, which is different from the finding by Gao et al in the human esophagus ${ }^{[17]}$. These results further demonstrate species difference of AQP1 expression in enteric nervous system. The physiological function of AQP1 as a water channel in the enteric nervous system remains unknown. Apparently, more systematic studies are required to clarify the function as well as the expression patterns of AQP1 in the enteric nervous systems in human and different species of animal models.

\section{Expression of AQP1 in the mechanoreceptive periodontal Ruffini endings}

Nandasena et al reported the immunolocalization of AQP1 in the periodontal Ruffini endings of the rat incisors and trigeminal ganglion ${ }^{[21]}$. AQP1 immunostaining was detected in the axon terminals of the periodontal Ruffini endings as well as their associated terminal Schwann cells, as confirmed with a double staining with AQP1 and either a neuronal marker PGP9.5 or a glial marker S-100 protein. In addition, the study determined AQP1 expression in about $16.1 \%$ trigeminal neurons and in certain satellite cells which surrounded AQP1positive or -negative neurons. An analysis of a cross-sectional area of these AQP1-positive neurons demonstrated that approximately $66.9 \%$ of the positive neurons were $400-1000$ $\mu^{2}$ (averaged at $671.4 \pm 172.4 \mu \mathrm{m}^{2}$ ), indicating that they belong to medium-sized neurons that mediate mechanotransduction. This is the first report confirming that the axon terminals of mechanoreceptors are positive for an AQP. Further investigation is needed for clarifying co-localization of SP/AQP1 or TRPV1/AQP1 in the trigeminal ganglion to determine the proportion of AQP1-positive neurons that are similar to dorsal root ganglion nociceptors. These findings suggest that AQP1 may control water transport in the periodontal Ruffini endings. But whether AQP1 is involved in neural signal transduction in the mechanoreceptive periodontal Ruffini endings remains to be determined.

\section{Expression and regulation of AQP2 in peripheral nervous system}

The first report about AQP2 expression in peripheral nervous system was seen in a study of AQP distribution in human tissue microarrays by Mobasheri et al in 2005 ${ }^{[22]}$. AQP2 was described to be expressed in peripheral nerve bundles. But no information about tissue origin was provided. In 2009, Radella's group reported two studies about regulated expression of AQP2 in rodent peripheral nerve and its possible involvement in pain transmission ${ }^{[23,24]}$.

The first study analyzed the expression of AQP2 in the trigeminal ganglia in an mouse model of perioral acute inflammatory pain induced by formalin ${ }^{[23]}$. The data showed altered AQP2 expression in trigeminal ganglia in acute inflammatory pain. In the control group, the AQP2 immunostaining showed general labeling in the cytoplasm of each neuronal class that appeared stronger in the medium- and large-sized neurons than in the small neurons. After formalin treatment, there was a marked increase of AQP2 expression in small-sized neurons and a decrease in medium- and large-sized neurons. A strong increase of AQP2 protein in the neuronal membrane of smallsized neurons was seen, suggesting increased expression and intracellular redistribution of AQP2 mainly in small-sized neurons. The expression pattern of AQP1 in the ganglia remained unaltered after formalin treatment. Quantitative immunoblot analysis also indicated increased expression of AQP2, but not AQP1 upon formalin treatment. These data support the hypothesis that AQP2 is involved in pain transmission in the peripheral nervous system.

In the second study, they analyzed the presence and localization of AQP2 in the spinal cord and dorsal root ganglia of normal rats and evaluated AQP2 expression in response to chronic constriction injury of the sciatic nerve, a model of neuropathic pain ${ }^{[24]}$. The results showed that although AQP2 expression was not detectable in the dorsal root ganglia of normal rats, a remarkable increase of AQP2 expression in response to chronic constriction injury treatment was seen in small-diameter neurons in dorsal root ganglia. These data suggested that AQP2 expression was involved in neuropathic nerve injuries, although its precise role remains to be determined.

The exact mechanism by which AQP2 is enhanced in DRG neurons is not clear. AQP2 is known as a regulated water channel both at protein translocation and transcriptional level in response to arginine vasopressin (AVP) in the renal collecting ducts through $\mathrm{V} 2$ receptor $^{[25]}$. AVP and its V1 receptor were reported to present in $\mathrm{DRG}^{[26,27]}$. It is not known whether AQP2 is regulated by AVP in DRG. Further studies are warranted to elucidate the mechanism of pain-induced AQP2 expression and function in peripheral sensory ganglia.

\section{Expression of AQP4 in peripheral nervous system}

Role of AQP4 in the central nervous system has been a major focus in the aquaporin field. Many important functions of AQP4 have been determined including brain edema formation 
and absorption, pathogenesis of neuromyelitis optica, hydrocephalus and pain perception ${ }^{[3-6,8-12,28]}$. Little is known about the expression and function of AQP4 in the peripheral nervous system.

In a recent study, Thi et al identified AQP4 protein expression in the myenteric and submucosal nerve plexuses of the mouse and the rat colon by immunofluorescence ${ }^{[29]}$. In the myenteric plexus, about $12 \%$ in the mouse and $13 \%$ in the rat myenteric neurons were AQP4 positive as revealed by double staining of the enteric ganglia with antibodies to AQP4 and the neuronal marker NF-H (neurofilament-heavy chain 100). In the submucosal plexus, nearly $80 \%$ neurons were positive for AQP4 in both the mouse and the rat colon. Double labeling for AQP4 and the glial marker GFAP (glial fibrillary acidic protein) verified that glial cells in the enteric plexuses were not immunoreactive to AQP4. In the same study, they confirmed a neuronal distribution of AQP1 in the myenteric and the submucosal plexuses similar to that reported by Nagahama et al in the rat small intestine ${ }^{[18]}$. This study clearly indicated that neurons rather than glial cells contained AQP4 in the colonic nerve plexuses, providing the first evidence that AQP4 was differentially expressed in peripheral versus central nervous system. The expression of AQP4 in myenteric and submucosal neurons suggests that AQP4 may be involved in intestinal motility and the neuronal regulation of transport processes in the intestinal mucosa. The researchers did not analyze the colocalization of AQP4 with neuronal markers specific to sensory neurons. Therefore, it is not known whether AQP4 is expressed in enteric sensory neurons.

Another recent study reported by Ablimit et al identified AQP4 expression in the neuronal sensory cells of the vomeronasal organ ${ }^{[30]}$. Vomeronasal organ is part of the nasal chemosensory system situated at the base of the nasal septum in the anterior nasal cavity ${ }^{[31,32]}$ and is anatomically and physiologically distinct from the olfactory system ${ }^{[33-35]}$. The sensory epithelium of the vomeronasal organ is composed of a sensory cell layer and supporting cell layer. AQP4 expression was highly concentrated in the sensory cells of the sensory epithelium. By immunogold electron microscopy, AQP4 protein was localized to the plasma membrane of neuronal sensory cells. Gold labeling representing AQP4 is clearly seen along the entire plasma membrane of the cell body and axons except for the apical membrane facing the surface of the lumen. In contrast to the presence of AQP4 in the axon forming the vomeronasal nerve, nerve fiber bundles running in the lamina propria of the nonsensory mucosa were negative for AQP4, suggesting a specific role of AQP4 in the neuronal transduction of the vomeronasal organ. A previous study indicated impaired olfaction in mice lacking AQP4 water channel ${ }^{[36]}$. Vomeronasal organ is regarded as a chemosensory organ for pheromones ${ }^{[34]}$. It will be interesting to study the role of AQP4 in the regulation of social behavior and sexual preference in AQP4 knockout mouse model.

\section{Perspectives}

The study on the expression and function of AQPs in the peripheral nervous system is still in an early stage although significant progress has been made in this field (summarized in Table 1). The picture on the distribution of AQP family members in the peripheral nervous system is far from complete. The precise cellular localization of AQPs in different types of peripheral nerves requires further systematic studies in human as well as model animals. The functions of AQPs in the peripheral nervous system are still largely unknown. The conflict results on the role of AQP1 in pain perception will await more investigations to clarify before AQP1 can become

Table 1. Expression and function of aquaporins in peripheral nervous system.

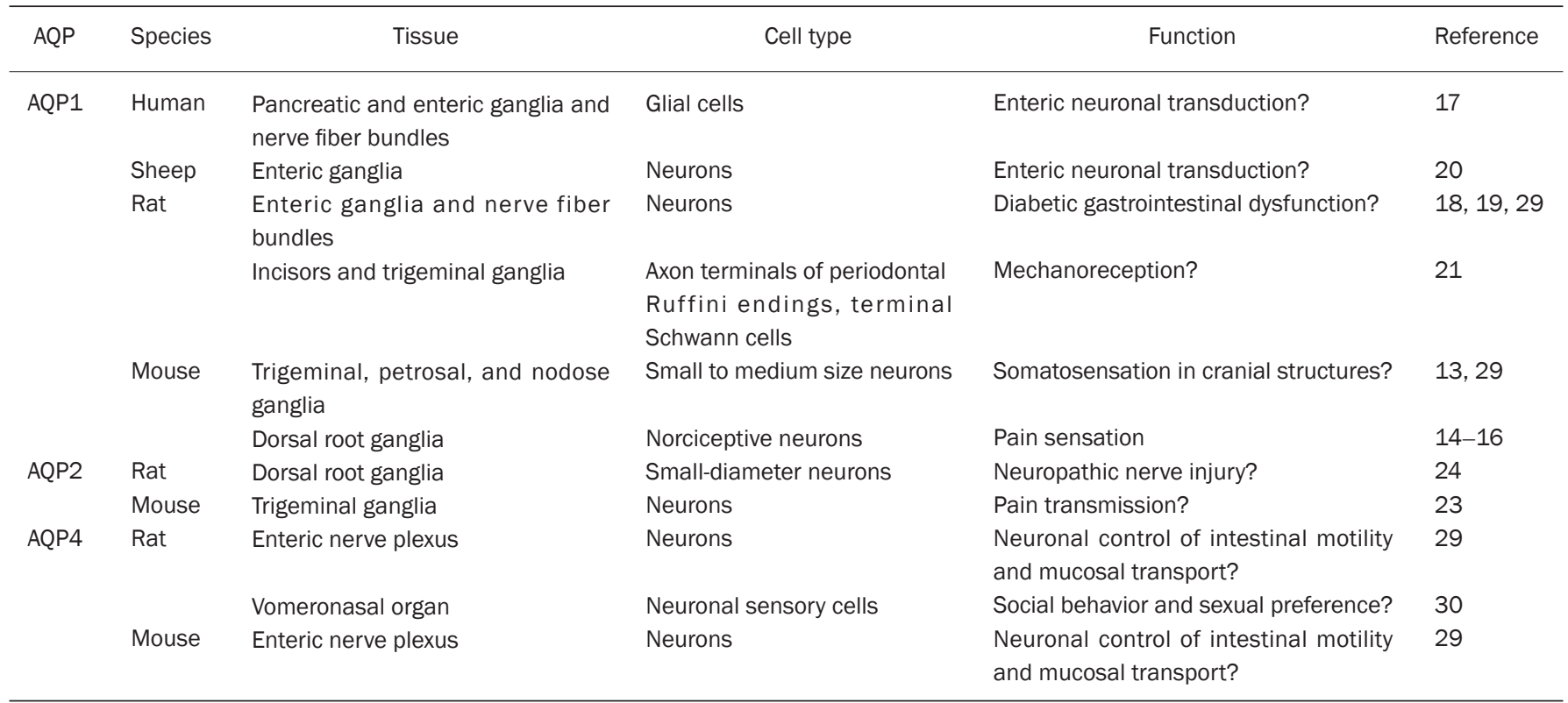


a drug target to reduce pain nociception. The physiological functions of AQP1 and AQP4 in enteric nerve plexuses remain to be determined. The role of AQP1 in mechanoreception requires further investigation. The mechanism of regulated AQP2 expression in neurons of peripheral sensory ganglia and its functional significance in pain transmission needs further elucidation. The physiological importance of AQP4 in sensory neurons of vomeronasal organ remains to be uncovered. More functional studies using transgenic AQP knockout mouse models or human subjects with lose-of-function mutations are required to establish the role of AQPs in the peripheral nervous system and evaluate their potential as novel drug targets for peripheral neuropathies.

\section{Acknowledgements}

This work was supported by National Natural Science Foundation (№ 30670477), Dalian Municipal Science and Technology Fund (2008E11SF162), and National Basic Research Program of China (“973” Program, № 2009CB521908).

\section{References}

1 Verkman AS. Mammalian aquaporins: diverse physiological roles and potential clinical significance. Expert Rev Mol Med 2008; 10: e13.

2 Carbrey JM, Agre P. Discovery of the aquaporins and development of the field. Handb Exp Pharmacol 2009; 190: 3-28.

3 Tait MJ, Saadoun S, Bell BA, Papadopoulos MC. Water movements in the brain: role of aquaporins. Trends Neurosci 2008; 31: 37-43.

4 Verkman AS, Binder DK, Bloch O, Auguste K, Papadopoulos MC. Three distinct roles of aquaporin-4 in brain function revealed by knockout mice. Biochim Biophys Acta 2006; 1758: 1085-93.

5 Zador Z, Stiver S, Wang V, Manley GT. Role of aquaporin-4 in cerebral edema and stroke. Handb Exp Pharmacol 2009; 190: 159-70.

6 Zheng GQ, Li Y, Gu Y, Chen XM, Zhou Y, Zhao SZ, et al. Beyond water channel: aquaporin-4 in adult neurogenesis. Neurochem Int 2010; 56: 651-4.

7 Jarius S, Wildemann B. AQP4 antibodies in neuromyelitis optica: diagnostic and pathogenetic relevance. Nat Rev Neurol 2010; 6: 383-92.

8 Nesic O, Guest JD, Zivadinovic D, Narayana PA, Herrera JJ, Grill RJ, et al. Aquaporins in spinal cord injury: the janus face of aquaporin 4 . Neuroscience 2010; 168: 1019-35.

9 Filippidis AS, Kalani MY, Rekate HL. Hydrocephalus and aquaporins: lessons learned from the bench. Childs Nerv Syst 2011; 27: 27-33.

10 Bao F, Chen M, Zhang Y, Zhao Z. Hypoalgesia in mice lacking aquaporin-4 water channels. Brain Res Bull 2010; 83: 298-303.

11 Chen ML, Bao F, Zhang YQ, Zhao ZQ. Effects of aquaporin 4 deficiency on morphine analgesia and chronic tolerance: a study at spinal level. J Mol Neurosci 2010; 42: 140-4.

12 Xu GY, Wang F, Jiang X, Tao J. Aquaporin 1, a potential therapeutic target for migraine with aura. Mol Pain 2010; 6: 68.

13 Matsumoto I, Nagamatsu N, Arai S, Emori Y, Abe K. Identification of candidate genes involved in somatosensory functions of cranial sensory ganglia. Brain Res Mol Brain Res 2004; 126: 98-102.

14 Oshio K, Watanabe H, Yan D, Verkman AS, Manley GT. Impaired pain sensation in mice lacking Aquaporin-1 water channels. Biochem Biophys Res Commun 2006; 341: 1022-8.

15 Shields SD, Mazario J, Skinner K, Basbaum Al. Anatomical and func- tional analysis of aquaporin 1, a water channel in primary afferent neurons. Pain 2007; 131: 8-20.

16 Zhang $\mathrm{H}$, Verkman AS. Aquaporin-1 tunes pain perception by interaction with $\mathrm{Na}(\mathrm{v}) 1.8 \mathrm{Na}^{+}$channels in dorsal root ganglion neurons. J Biol Chem 2010; 285: 5896-906.

17 Gao H, He C, Fang X, Hou X, Feng X, Yang H, et al. Localization of aquaporin-1 water channel in glial cells of the human peripheral nervous system. Glia 2006; 53: 783-7.

18 Nagahama M, Ma N, Semba R, Naruse S. Aquaporin 1 immunoreactive enteric neurons in the rat ileum. Neurosci Lett 2006; 395: 206-10.

19 Ishihara E, Nagahama M, Naruse S, Semba R, Miura T, Usami M, et al. Neuropathological alteration of aquaporin 1 immunoreactive enteric neurons in the streptozotocin-induced diabetic rats. Auton Neurosci 2008; 138: 31-40.

20 Arciszewski MB, Stefaniak M, Zacharko-Siembida A, Całka J. Aquaporin 1 water channel is expressed on submucosal but not myenteric neurons from the ovine duodenum. Ann Anat 2011; 193: 81-5.

21 Nandasena BG, Suzuki A, Aita M, Kawano Y, Nozawa-Inoue K, Maeda T. Immunolocalization of aquaporin-1 in the mechanoreceptive Ruffini endings in the periodontal ligament. Brain Res 2007; 1157: 32-40.

22 Mobasheri A, Wray S, Marples D. Distribution of AQP2 and AQP3 water channels in human tissue microarrays. J Mol Histol 2005; 36: $1-14$.

23 Borsani E, Bernardi S, Albertini R, Rezzani R, Rodella LF. Alterations of AQP2 expression in trigeminal ganglia in a murine inflammation model. Neurosci Lett 2009; 449: 183-8.

24 Buffoli B, Borsani E, Rezzani R, Rodella LF. Chronic constriction injury induces aquaporin-2 expression in the dorsal root ganglia of rats. J Anat 2009; 215: 498-505.

25 Kwon TH, Hager H, Nejsum LN, Andersen ML, Frokiaer J, Nielsen S. Physiology and pathophysiology of renal aquaporins. Semin Nephrol 2001; 21: 231-8.

26 Horn AM, Lightman SL. Vasopressin-induced turnover of phosphatidylinositol in the sensory nervous system of the rat. Exp Brain Res 1987; 68: 299-304.

27 Ma KK, Che YM. Distribution of arginine-vasopressin in the trigeminal, dorsal root ganglia and spinal cord of the rat; depletion by capsaicin. Comp Biochem Physiol A Physiol 1995; 110: 71-8.

28 Verkman AS. Aquaporins: translating bench research to human disease. J Exp Biol 2009; 212: 1707-15.

29 Thi MM, Spray DC, Hanani M. Aquaporin-4 water channels in enteric neurons. J Neurosci Res 2008; 86: 448-56.

30 Ablimit A, Aoki T, Matsuzaki T, Suzuki T, Hagiwara H, Takami S, et al. Immunolocalization of water channel aquaporins in the vomeronasal organ of the rat: expression of AQP4 in neuronal sensory cells. Chem Senses 2008; 33: 481-8.

31 Døving KB, Trotier D. Structure and function of the vomeronasal organ. J Exp Biol 1998; 201: 2913-25.

32 Takami S. Recent progress in the neurobiology of the vomeronasal organ. Microsc Res Tech 2002; 58: 228-50.

33 Halpern M, Jia C, Shapiro LS. Segregated pathways in the vomeronasal system. Microsc Res Tech 1998; 41: 519-29.

34 Witt M, Woźniak W. Structure and function of the vomeronasal organ. Adv Otorhinolaryngol 2006; 63: 70-83.

35 Martinez-Marcos A. On the organization of olfactory and vomeronasal cortices. Prog Neurobiol 2009; 87: 21-30.

36 Lu DC, Zhang H, Zador Z, Verkman AS. Impaired olfaction in mice lacking AQP4 water channels. FASEB J 2008; 22: 3216-23 